\title{
TSPAN8 and distant metastasis of nasopharyngeal carcinoma cells
}

\author{
Changqing Xie, Nicole A. McGrath
}

Thoracic and GI Malignancies Branch, Center for Cancer Research, National Cancer Institute, National Institutes of Health, Bethesda, MD, USA

Correspondence to: Changqing Xie, MD, PhD. National Cancer Institute, 9000 Rockville Pike, 10/3B43C, Bethesda, MD 20892 , USA.

Email: changqing.xie@nih.gov.

Provenance and Peer Review: This article was commissioned by the Editorial Office, Annals of Translational Medicine. The article did not undergo external peer review.

Comment on: Lin X, Bi Z, Hu Q, et al. TSPAN8 serves as a prognostic marker involving Akt/MAPK pathway in nasopharyngeal carcinoma. Ann Transl Med 2019;7:470.

Submitted Oct 16, 2019. Accepted for publication Oct 29, 2019.

doi: 10.21037/atm.2019.10.102

View this article at: http://dx.doi.org/10.21037/atm.2019.10.102

Nasopharyngeal carcinoma (NPC) is an aggressive epithelial carcinoma that is prevalent in Southeast Asia, Southern China and North Africa. It can develop in the presence of various risk factors, including Epstein-Barr virus (EBV) infection, environmental exposure to carcinogens, ethnic background, and genetic predisposition. It is prone to local invasion and early distant metastasis. Patients with earlystage NPC have a relatively high cure rate of over $90 \%$ with radical radiotherapy. In contrast, the 5-year overall survival (OS) rate for locally advanced NPC declines to between $50-60 \%$ (1). With the combination of intensity-modulated radiotherapy, chemotherapy, surgery and targeted therapy, the locoregional control rate has been reported to be 80-90\% in locally advanced NPC, whereas distant metastasis remains the major reason of treatment failure in these patients (2-4). The outcome for patients with distant metastatic NPC is suboptimal, with a median OS of 20 months (5). Unfortunately, roughly 15\% NPC patients present with distant metastases at their first diagnosis (6). The understanding of the mechanisms that drive NPC metastasis will be substantially beneficial for developing new and reliable biomarkers for early detection and novel therapeutic strategies for NPC.

The specific molecular mechanisms that drive NPC metastasis remain unclear. The most common histologic type in Eastern countries is undifferentiated carcinoma, while squamous cell carcinoma is more common in USA and Europe. Undifferentiated carcinoma is strongly associated with EBV infection. EBV encodes the oncogene product, latent membrane protein 1 (LMP1). LMP1 executes its oncogenic functions via activating the P38 MAPK signaling pathway, and subsequently decreasing the sensitivity of NPC cells to ionizing radiation (7). Moreover, LMP1-mediated metabolic reprogramming activates IGF1-mTORC2 signaling pathway, facilitates PDHE1 $\alpha$ nuclear translocation that leads to acetylation and activation of the Snail promoter (8). However, no efficient EBV targeted therapy has been developed in NPC treatment. Intriguingly, noncoding RNAs, including microRNAs and long noncoding RNAs, are increasingly implicated and appreciated as playing critical roles in the mediation of NPC metastasis (9-12).

In this Journal, Lin et al. reported an association between Tetraspanin 8 (TSPAN8) and distant metastasis of NPC (13). There was 1,787 differential expressed genes between paired tumor tissues and benign adjacent tissues from NPC with 8 genes that were highly upregulated in NPC tissues. However, only TSPAN8 is over-expressed in the poorly differentiated CNE2 cell line and the highly metastatic subclone S18 NPC cell line. More importantly, TSPAN8 promotes invasion and migration in NPC cell lines in vitro. When TSPAN8 is silenced in poorly differentiated CNE2 cells, it leads to the down-regulation of proinflammatory factor IL-1 $\beta$, which inhibits the AKT/MAPK pathway and attenuates metastasis. The authors further explored whether TSPAN8 could predict the prognosis of NPC. Immunohistochemistry experiments indicated that increased TSPAN8 level in NPC was linked to short OS 
and metastasis-free survival, suggesting that TSPAN8 could be utilized as a prognostic biomarker for NPC patients (13). This is the first report to suggest that TSPAN8 plays a critical role in the progression and metastasis of NPC.

The TSPAN8 gene encodes for a cell surface glycoprotein that is a member of the 4-transmembrane protein family. It was originally found to be expressed in several types of cancers but not in most normal tissues (14). Subsequently, it was found to be involved in the progression of pancreatic cancer (15), breast cancer (16), lung cancer (17), melanoma $(18,19)$, gastric cancer (20) and hepatocellular cancer (21). It was also discovered that $\beta$-catenin stabilization is a molecular response after the onset of TSPAN8 activation in melanoma, that suggests that $\beta$-catenin and TSPAN8 are part of a positive feedback loop and sustains a high TSPAN8 expression level (19). The knockout of TSPAN8 down-regulates WNT pathway activity, reduces $\beta$-catenin expression and subsequent translocation to the nucleus in gastric cancer (22). The effect of TSPAN8 on $\beta$-catenin is mediated by the binding to NOTCH2 (22). In addition, TSPAN8 promotes gastric cancer cell growth and metastasis at least partially through the activation of ERK-MAPK pathway (23). Furthermore, it has been shown that TSPAN8 and its regulators control early melanoma invasion. This indicates that TSPAN8 is a promising novel therapeutic target by regulating downstream of the RAF-MEK-ERK signaling pathway (18). However, it is unknown if the function of TSPAN8 in NPC is associated with EBV virus status. This topic remains one of interest given the prevalence of EBV infection in patients with NPC. Notably, the authors of this study have demonstrated that TSPAN8 may play a role in tumor progression and metastasis through AKT/MAPK pathway in NPC, which implicates a new mechanism of TSPAN8 in regulating cancer cells.

Interestingly, the role of TSPAN8 in promoting cancer stemness has been highlighted recently $(16,24,25)$. Cancer stem cells (CSCs) are a small cell population within the tumor microenvironment (TME). Emerging evidence has suggested that CSCs serve as the basis of cancer metastasis, solid tumor progression, and therapeutic resistance. TSPAN8 has been used as a marker of CSCs and was found to promote cancer stemness through regulating stemness genes: NANOG, OCT4, and ALDHA1 $(16,24)$. It is also an important exosome component to mediate crosstalk between CSCs and their neighboring cells (25). Furthermore, the expression of TSPAN8 is upregulated in breast CSCs and enhances stemness maintenance through the activation of Hedgehog signaling (16). Therefore, it is suggested that TSPAN8 is a potential therapeutic target to overcome treatment resistance contributed by CSCs. It remains undecided that TSPAN8 contributes to distant metastasis and treatment failure in NPC by functioning as CSC stemness guide. It would be worthy to further explore this in order to develop effective therapeutic target to control distant metastasis.

Collectively, this study has shown that TSPAN8 promotes NPC progression and metastasis through AKT/ MAPK pathway. TSPAN8 is a potential biomarker for predicting metastasis and prognosis of NPC patients, as well as a therapeutic target for NPC treatment.

\section{Acknowledgments}

Funding: None.

\section{Footnotes}

Conflicts of Interest: The authors have no conflicts of interest to declare.

Ethical Statement: The authors are accountable for all aspects of the work in ensuring that questions related to the accuracy or integrity of any part of the work are appropriately investigated and resolved.

Open Access Statement: This is an Open Access article distributed in accordance with the Creative Commons Attribution-NonCommercial-NoDerivs 4.0 International License (CC BY-NC-ND 4.0), which permits the noncommercial replication and distribution of the article with the strict proviso that no changes or edits are made and the original work is properly cited (including links to both the formal publication through the relevant DOI and the license). See: https://creativecommons.org/licenses/by-nc-nd/4.0/.

\section{References}

1. Lee AW, Poon YF, Foo W, et al. Retrospective analysis of 5037 patients with nasopharyngeal carcinoma treated during 1976-1985: overall survival and patterns of failure. Int J Radiat Oncol Biol Phys 1992;23:261-70.

2. Chen YP, Chan ATC, Le QT, et al. Nasopharyngeal carcinoma. Lancet 2019;394:64-80.

3. Pan JJ, Ng WT, Zong JF, et al. Proposal for the 8th edition of the AJCC/UICC staging system for nasopharyngeal 
cancer in the era of intensity-modulated radiotherapy. Cancer 2016;122:546-58.

4. Peng G, Wang T, Yang KY, et al. A prospective, randomized study comparing outcomes and toxicities of intensity-modulated radiotherapy vs. conventional two-dimensional radiotherapy for the treatment of nasopharyngeal carcinoma. Radiother Oncol 2012;104:286-93.

5. Wei WI, Sham JS. Nasopharyngeal carcinoma. Lancet 2005;365:2041-54.

6. Tang LQ, Chen QY, Fan W, et al. Prospective study of tailoring whole-body dual-modality [18F] fluorodeoxyglucose positron emission tomography/ computed tomography with plasma Epstein-Barr virus DNA for detecting distant metastasis in endemic nasopharyngeal carcinoma at initial staging. J Clin Oncol 2013;31:2861-9.

7. Zhang Z, Yu X, Zhou Z, et al. LMP1-positive extracellular vesicles promote radioresistance in nasopharyngeal carcinoma cells through P38 MAPK signaling. Cancer Med 2019;8:6082-94.

8. Zhang J, Jia L, Liu T, et al. mTORC2-mediated PDHE1alpha nuclear translocation links EBV-LMP1 reprogrammed glucose metabolism to cancer metastasis in nasopharyngeal carcinoma. Oncogene 2019;38:4669-84.

9. Zhang P, Lu X, Shi Z, et al. miR-205-5p regulates epithelial-mesenchymal transition by targeting PTEN via PI3K/AKT signaling pathway in cisplatin-resistant nasopharyngeal carcinoma cells. Gene 2019;710:103-13.

10. Hua X, Fan KC. Down-regulation of miR-1181 indicates a dismal prognosis for nasopharyngeal carcinoma and promoted cell proliferation and metastasis by modulating Wnt/beta-catenin signaling. Eur Rev Med Pharmacol Sci 2019;23:1077-86.

11. Hu X, Liu W, Jiang X, et al. Long noncoding RNA LINC00460 aggravates invasion and metastasis by targeting miR-30a-3p/Rap1A in nasopharyngeal carcinoma. Hum Cell 2019;32:465-76.

12. Zhang W, Wang L, Zheng F, et al. Long Noncoding RNA Expression Signatures of Metastatic Nasopharyngeal Carcinoma and Their Prognostic Value. Biomed Res Int 2015;2015:618924.

13. Lin X, Bi Z, Hu Q, et al. TSPAN8 serves as a prognostic marker involving Akt/MAPK pathway in nasopharyngeal carcinoma. Ann Transl Med 2019; 7:470.

14. Szala S, Kasai Y, Steplewski Z, et al. Molecular cloning of
cDNA for the human tumor-associated antigen CO-029 and identification of related transmembrane antigens. Proc Natl Acad Sci U S A 1990;87:6833-7.

15. Wang Z, Sun H, Provaznik J, et al. Pancreatic cancerinitiating cell exosome message transfer into noncancerinitiating cells: the importance of CD44v6 in reprogramming. J Exp Clin Cancer Res 2019;38:132.

16. Zhu R, Gires O, Zhu L, et al. TSPAN8 promotes cancer cell stemness via activation of sonic Hedgehog signaling. Nat Commun 2019;10:2863.

17. Li Y, Xiao X, Bosse Y, et al. Genetic interaction analysis among oncogenesis-related genes revealed novel genes and networks in lung cancer development. Oncotarget 2019;10:1760-74.

18. Agaesse G, Barbollat-Boutrand L, Sulpice E, et al. A largescale RNAi screen identifies LCMR1 as a critical regulator of Tspan8-mediated melanoma invasion. Oncogene 2017;36:5084.

19. El Kharbili M, Agaesse G, Barbollat-Boutrand L, et al. Tspan8-beta-catenin positive feedback loop promotes melanoma invasion. Oncogene 2019;38:3781-93.

20. Lin H, Zhou AJ, Zhang JY, et al. MiR-324-5p reduces viability and induces apoptosis in gastric cancer cells through modulating TSPAN8. J Pharm Pharmacol 2018;70:1513-20.

21. Fang T, Lin J, Wang Y, et al. Tetraspanin-8 promotes hepatocellular carcinoma metastasis by increasing ADAM12m expression. Oncotarget 2016;7:40630-43.

22. Li L, Yang D, Cui D, et al. Quantitative proteomics analysis of the role of tetraspanin-8 in the drug resistance of gastric cancer. Int J Oncol 2018;52:473-84.

23. Wei L, Li Y, Suo Z. TSPAN8 promotes gastric cancer growth and metastasis via ERK MAPK pathway. Int J Clin Exp Med 2015;8:8599-607.

24. Mutoji K, Singh A, Nguyen T, et al. TSPAN8 Expression Distinguishes Spermatogonial Stem Cells in the Prepubertal Mouse Testis. Biol Reprod 2016;95:117.

25. Yue $\mathrm{S}, \mathrm{Mu} W$, Erb U, et al. The tetraspanins CD151 and Tspan 8 are essential exosome components for the crosstalk between cancer initiating cells and their surrounding. Oncotarget 2015;6:2366-84.

Cite this article as: Xie C, McGrath NA. TSPAN8 and distant metastasis of nasopharyngeal carcinoma cells. Ann Transl Med 2020;8(5):165. doi: 10.21037/atm.2019.10.102 